\title{
Auriculoterapia no controle dos sintomas da ansiedade de profissionais de saúde: uma revisão sistemática
}

Auriculotherapy in the control of anxiety symptoms in health personnel: a systematic review

Auriculoterapia en el control de los síntomas de ansiedad en profesionales de la salud: una revisión sistemática

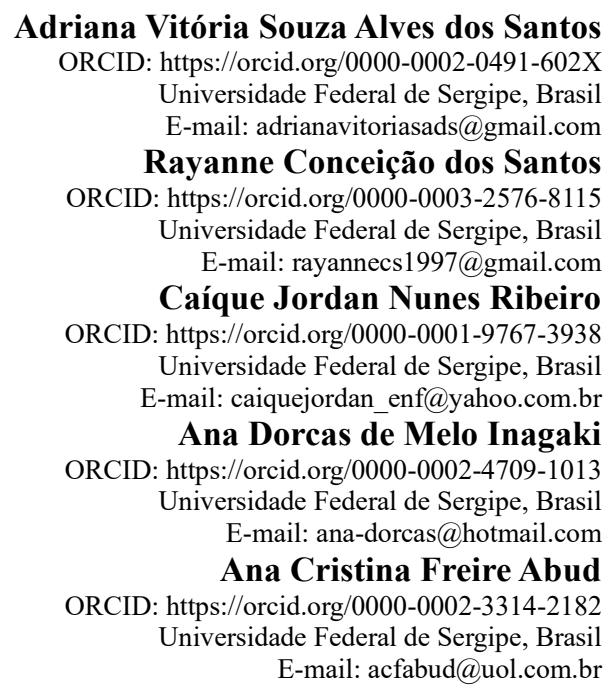

\begin{abstract}
Resumo
A ansiedade é um problema comum entre os profissionais da saúde, no entanto, poucos estudos investigam métodos não farmacológicos, como a auriculoterapia, para o seu controle. O objetivo do estudo foi realizar uma revisão sistemática sobre a eficácia da auriculoterapia no controle dos sintomas da ansiedade de profissionais de saúde. Foram seguidas as recomendações do Preferred Reporting Items for Systematic Reviews and Meta-analyses 2020 (PRISMA 2020). Foram incluídos ensaios controlados randomizados nos idiomas português, inglês e espanhol, sem restrição de ano de publicação, registrados nas bases de dados MEDLINE/PubMed, Bireme, Scopus, Embase, CENTRAL (Cochrane Library) e Google Acadêmico. Dos 213 registros encontrados, um ensaio clínico realizado no Brasil foi incluído na análise final. Os resultados demonstraram que a auriculoterapia reduziu os sintomas de ansiedade em 17\% no grupo tratado com agulhas, $13 \%$ com sementes e $11 \%$ com fitas adesivas. Dessa forma, embora a auriculoterapia tenha contribuído para o controle dos sintomas da ansiedade em profissionais de saúde, as evidências são escassas.
\end{abstract}

Palavras-chave: Auriculoterapia; Ansiedade; Profissionais de saúde; Medicina tradicional chinesa.

\begin{abstract}
Anxiety is a common problem among health professionals, however, few studies investigate non-pharmacological methods such as auriculotherapy for its control. The aim of the study was to carry out a systematic review of the effectiveness of auriculotherapy in the control of anxiety symptoms in healthcare professionals. The recommendations of the Preferred Reporting Items for Systematic Reviews and Meta-analyses 2020 (PRISMA 2020) were followed. Randomized controlled trials in Portuguese, English and Spanish were included, without restriction of year of publication, registered in the MEDLINE/PubMed, Bireme, Scopus, Embase, CENTRAL (Cochrane Library) and Academic Google databases. Of the 213 records found, one clinical trial carried out in Brazil was included in the final analysis. The results showed that auriculotherapy reduced anxiety symptoms by $17 \%$ in the group treated with needles, $13 \%$ with seeds and $11 \%$ with adhesive tape. Thus, although auriculotherapy has contributed to the control of anxiety symptoms in health professionals, evidence is scarce.
\end{abstract}

Keywords: Auriculotherapy; Anxiety; Health professionals; Traditional chinese medicine. 


\section{Resumen}

A pesar de que la ansiedad es un problema común entre los profesionales de la salud, pocos estudios han investigado métodos no farmacológicos, como la auriculoterapia, para su control. El objetivo del estudio fue realizar una revisión sistemática de la efectividad de la auriculoterapia que no controla dos síntomas de ansiedad que experimentan los profesionales de la salud. Se siguieron como recomendaciones los elementos de informes preferidos para revisiones sistemáticas y metaanálisis 2020 (PRISMA 2020). Se incluyeron ensayos clínicos aleatorizados en portugués, inglés y español, con restricción de año de publicación, registrados en las bases de datos MEDLINE / PubMed, Bireme, Scopus, Embase, CENTRAL (Cochrane Library) y Google Academic. En el análisis final se incluyeron dos 213 historias clínicas encontradas, un ensayo clínico realizado en Brasil. Los resultados muestran que la terapia auditiva redujo los síntomas de ansiedad en el $17 \%$ del grupo tratado con agujas, el 13\% con trastornos mentales y el 11\% con aditivos. Por lo tanto, la terapia auricular ha contribuido a controlar dos síntomas de ansiedad en la profesión de la salud, además de eventos raros.

Palabras clave: Auriculoterapia; Ansiedad; Profesionales de la salud; Medicina tradicional china.

\section{Introdução}

A ansiedade é uma condição natural do ser humano, porém, em excesso acaba por sobrecarregar o indivíduo, levando tanto a manifestações físicas, como tremor, sudorese e taquicardia; quanto a psicológicas, a exemplo da improdutividade, compulsão e fobias (Leão, Gomes, Ferreira \& Cavalcanti, 2018).

Em 2017, estimou-se que mais de 3,6\% da população mundial sofria com desordem decorrente da ansiedade, e as maiores taxas são encontradas no continente americano. Os profissionais da saúde constituem um grupo que rotineiramente está exposto a situações desgastantes, tais como: a necessidade de agilidade técnica, capacidade de liderança e resolução de problemas. Diante disso, o despreparo emocional, a sobrecarga de trabalho, o subdimensionamento de recursos humanos e materiais são desencadeadores do estresse e da ansiedade, os quais impactam no seu desempenho profissional e na sua vida pessoal (Costa et al. 2019; Moura et al., 2018; Vendemiatti et al., 2010).

Nesse contexto, as repercussões da ansiedade associadas ao fácil acesso a medicamentos, tornam os profissionais de saúde vulneráveis à automedicação e ao abuso de psicofármacos. Fato que pode resultar na maior incidência de erros, baixo rendimento, dificuldade de interação com a equipe, tolerância medicamentosa, dependência e óbito (Dantas, 2021; Minas, Rodacoski \& Sdoukos, 2019; Ribeiro et al., 2020).

Atualmente, com o enfrentamento da pandemia da COVID-19, gerou uma sobrecarga de trabalho desses profissionais, repercutindo no aumento de transtornos de saúde mental, sobretudo a ansiedade. Ressalta-se que, mundialmente, a prevalência da ansiedade entre profissionais de saúde durante a pandemia é de aproximadamente 31,9\% (Silva et al, 2021).

Diante desse cenário, as práticas integrativas e complementares (PICs) podem ser importantes aliadas no manejo de sintomas desse transtorno. A Organização Mundial da Saúde (OMS) recomenda o uso de medicina complementar desde 1978. No Brasil, as PICs são certificadas em uma política do Sistema Único de Saúde (SUS) (Moura et al., 2019).

Dentre essas práticas, pode-se destacar a auriculoterapia, uma vez que promove o alívio dos sintomas da ansiedade em adultos e idosos, não causa dependência, apresenta baixo custo e poucos efeitos colaterais, como a dor ou o incômodo no pavilhão auricular. Ademais, devido à praticidade de aplicação, a auriculoterapia pode atuar como medida adjuvante eficaz para a promoção e recuperação da saúde (Artioli, Tavares \& Bertolini, 2019; Corrêa et al., 2020; Moura et al., 2019).

Trata-se de um microssistema da acupuntura, método não farmacológico, realizado por meio do estímulo de partes do corpo representadas no pavilhão auricular. O estímulo dos acupontos tem o objetivo de tratar pessoas de forma integral, por repercutir nos pares de nervos cranianos, ao promover a homeostasia corporal e o relaxamento da mente e do corpo (Cardozobatista \& Tucci, 2020; Kwon, Lee \& Kim, 2020). 
A eficácia da auriculoterapia tem sido evidenciada em diferentes contextos, a saber: controle de peso em pacientes obesos; alívio da dor em pacientes com resistência às terapias farmacológicas; e tratamento adjuvante para a redução da pressão arterial (Asher et al., 2010; Corrêa et al., 2020; Freitas, Sousa \& Coutinho, 2020; Gao et al., 2019).

Nessa direção, estudos que investiguem estratégias para o alívio do transtorno de ansidade entre profissionais de saúde são necessários, uma vez que o sistema de saúde está cada vez mais complexo e a precarização das condições de trabalho expõe os profissionais à jornadas de trabalho exaustivas (Dias, Souza, Penna \& Gallasch, 2019). Portanto, o objetivo desse estudo foi realizar uma revisão sistemática sobre a eficácia da auriculoterapia no controle dos sintomas da ansiedade em profissionais de saúde.

\section{Metodologia}

Trata-se de um estudo de revisão sistemática que seguiu as recomendações do Preferred Reporting Items for Systematic Reviews and Meta-analyses 2020 (PRISMA 2020). Os elementos do acrônimo PICOT (population, intervention, comparator, outcome e type of study) foram utilizados para direcionar a questão de pesquisa e a estratégia de busca desta revisão:

$\mathbf{P}$ (população): profissionais da área da saúde;

I (intervenção): auriculoacupuntura, incluindo semente, cristais radiônicos, moxabustão, sangria, agulhas, eletroacupuntura, agulha de fogo, agulha de flor de ameixa e demais modalidades;

C (comparação): placebo, auriculoacupuntura simulada (sham), métodos farmacológicos e outras PICs;

O (desfecho): ansiedade;

T (tipo de estudo): ensaio clínico randomizado.

Diante do exposto, a presente revisão foi guiada pela seguinte questão norteadora: a auriculoterapia é eficaz no controle dos sintomas da ansiedade em profissionais de saúde?

Foram incluídos ensaios controlados randomizados que testaram a eficácia da auriculoacupuntura no controle da ansiedade em profissionais de saúde, nos idiomas português, inglês e espanhol e não houve restrição no ano de publicação. Estudos e séries de casos, editoriais, cartas ao editor, comentários, relatos de experiência, estudos observacionais (transversais, de coorte, de caso-controle e aninhados), revisões (narrativas, integrativas, de escopo e sistemáticas) e estudos envolvendo combinações de múltiplas PICs no grupo intervenção foram excluídos.

A busca foi realizada por dois revisores independentes em cinco bases de dados: MEDLINE/Pubmed (Medical Literature Analysis and Retrieval System Online), BIREME (Biblioteca Regional de Medicina), Embase, Scopus e Cochrane Central Register of Controlled Trials (CENTRAL). A literatura cinza foi investigada no motor de busca do Google Acadêmico e nas listas de referências dos artigos incluídos na leitura na íntegra. Foi realizada combinação de descritores controlados e de palavras-chave com operadores booleanos AND e OR. No Quadro 1 estão contidas as estratégias de busca nas respectivas bases de dados.

Inicialmente, os revisores realizaram a triagem dos estudos com base nos seus títulos e resumos. Os estudos considerados relevantes foram lidos na íntegra e selecionados de acordo com os critérios de elegibilidade. As discordâncias foram resolvidas por consenso ou por um terceiro revisor. Artigos indexados em mais de uma base de dados foram computados apenas uma vez.

A extração dos dados publicados foi realizada em uma planilha eletrônica padronizada pelos autores. Foram obtidas as seguintes variáveis: primeiro autor, periódico, ano de publicação, país, local do estudo (hospital, clínica, ambulatório, serviço pré-hospitalar ou unidade básica), população estudada (médicos, equipe de enfermagem, fisioterapeutas, nutricionistas, psicológos, odontólogos, terapeutas ocupacionais, profissionais de educação física e fonoaudiológos), tamanho da amostra, 
características dos participantes (idade, sexo, cor da pele, escolaridade, jornada de trabalho, comorbidades, tratamentos farmacológicos concomitantes), sintomas de ansiedade, protocolo da intervenção (quantidade e duração de sessões, pontos de aplicação e materiais utilizados), e desfecho avaliado (escala de mensuração).

Em relação à avaliação da qualidade metodológica dos estudos foi utilizada a ferramenta Risk of bias da Cochrane Colaboration. Esse instrumento possibilita a avaliação do risco de viés em sete domínios: geração de sequência e distribuição de alocação (viés de seleção), cegamento de participantes e pessoal (viés de desempenho), cegamento de avaliação de resultados (viés de detecção), dados de resultados incompletos (viés de atrito), relatos de resultados seletivos (viés de relato) e outras fontes potenciais de viés. O risco de viés foi classificado como alto, baixo e incerto (Carvalho, Silva \& Grande, 2013).

Quadro 1. Estratégias de busca.

\begin{tabular}{|c|c|}
\hline Base de Dados & Estratégia de Busca \\
\hline MEDLINE/ PubMed & $\begin{array}{l}\text { (nurse OR doctor OR physiotherapist OR pharmaceutical OR psychologist OR } \\
\text { speech therapist OR nutritionist OR physical education professional) [All fields] } \\
\text { AND ("acupuncture, ear" OR auriculotherapy) [All fields] AND (anxiety) [All } \\
\text { fields]. }\end{array}$ \\
\hline Bireme & ansiedade AND profissionais de saúde AND acupuntura auricular \\
\hline Embase & $\begin{array}{l}\text { (Nurse [All fields] OR doctor [All fields] OR physiotherapist [All fields] OR } \\
\text { pharmaceutical [All fields] OR psychologist [All fields] OR speech therapist [All } \\
\text { fields] OR nutritionist [All fields] OR physical education professional [All fields]) } \\
\text { AND ('auricular acupuncture' [All fields]) AND "anxiety" [All fields]. }\end{array}$ \\
\hline Scopus & $\begin{array}{l}\text { ("nurse" [All fields] OR "doctor" [All fields] OR "physiotherapist" [All fields] OR } \\
\text { "pharmaceutical" [All fields] OR "psychologist" [All fields] OR "speech therapist" } \\
\text { [All fields] OR "nutritionist" [All fields] OR "physical education professional" } \\
\text { [All fields]) AND ("acupuncture, ear" [All fields] OR ("auriculotherapy" [All } \\
\text { fields]) AND "anxiety" [All fields]. }\end{array}$ \\
\hline \multirow[b]{2}{*}{ Cochrane Library } & $\begin{array}{l}\text { ("nurse" [All fields] OR "doctor" [All fields] OR "physiotherapist" [All fields] OR } \\
\text { "pharmaceutical" [All fields] OR "psychologist" [All fields] OR "speech therapist" } \\
\text { [All fields] OR "nutritionist" [All fields] OR "physical education professional" } \\
\text { [All fields]) AND ("Acupuncture auricular" [All fields]) AND ("anxiety" [All } \\
\text { fields]) }\end{array}$ \\
\hline & $\begin{array}{l}\text { ("nurse" [All fields] OR “doctor" [All fields] OR "physiotherapist" [All fields] OR } \\
\text { "pharmaceutical" [All fields] OR "psychologist" [All fields] OR "speech therapist" } \\
\text { [All fields] OR "nutritionist" [All fields] OR "physical education professional" } \\
\text { [All fields]) AND ("acupuncture, ear" [All fields] OR ("auriculotherapy" [All } \\
\text { fields]) AND "anxiety" [All fields]. }\end{array}$ \\
\hline Google Acadêmico & $\begin{array}{l}\text { (nurse OR doctor OR physiotherapist OR pharmaceutical OR psychologist OR } \\
\text { speech therapist OR nutritionist OR physical education professional) AND } \\
\text { ("acupuncture, ear" OR auriculotherapy) AND (anxiety). }\end{array}$ \\
\hline
\end{tabular}

Fonte: Autores.

\section{Resultados}

A busca nas bases de dados resultou em 213 artigos ou estudos, dos quais cinco foram lidos na íntegra para análise dos critérios de inclusão e um foi incluído na análise final, conforme demonstrado na Figura 1. 
Figura 1. Fluxograma PRISMA de seleção dos estudos.

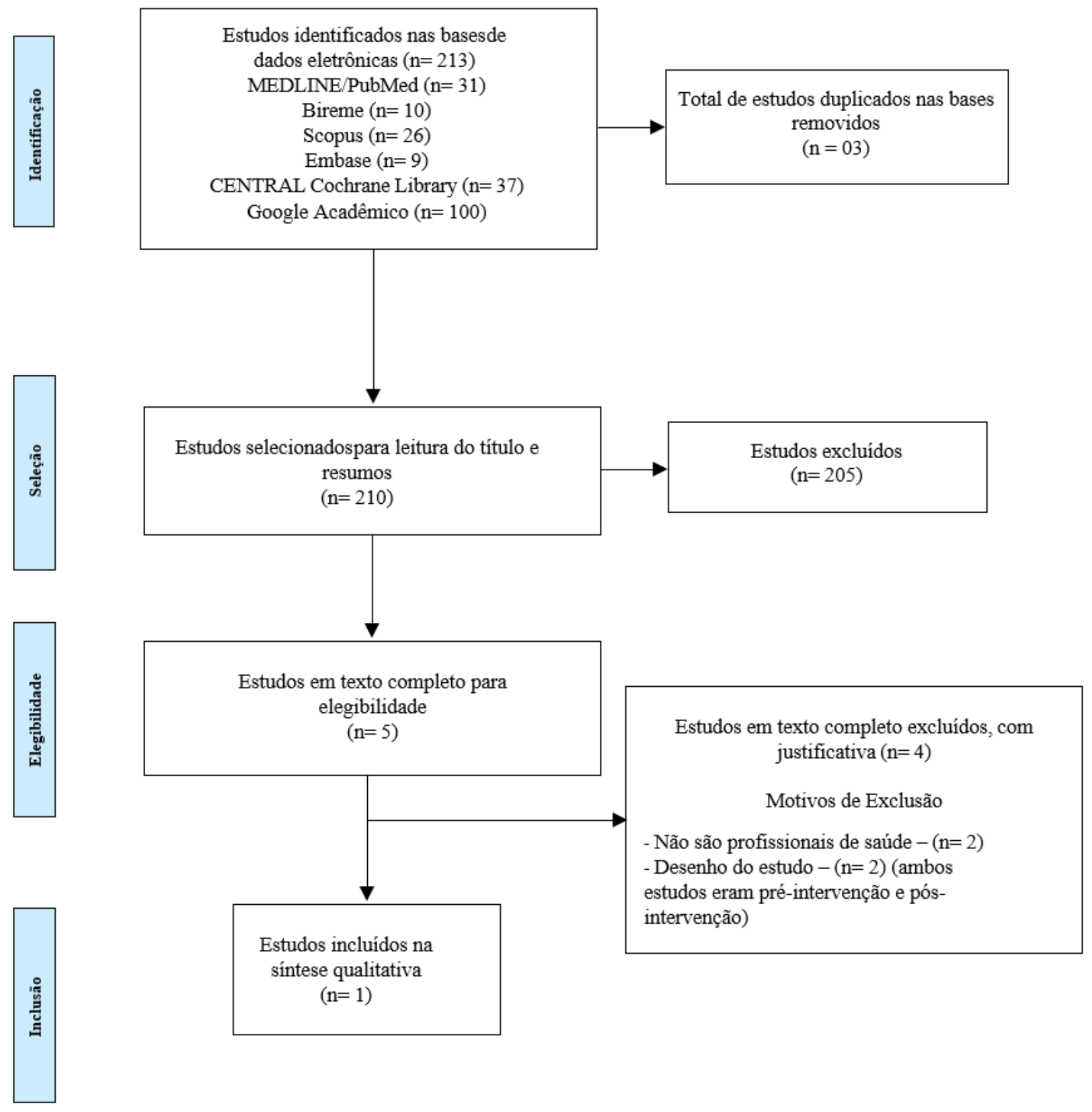

Fonte: Autores.

O ensaio que atendeu aos critérios de elegibilidade desta revisão foi publicado em inglês e desenvolvido no Brasil em 2017. O Quadro 2 apresenta suas características principais. 
Research, Society and Development, v. 10, n. 10, e153101018528, 2021

(CC BY 4.0) | ISSN 2525-3409 | DOI: http://dx.doi.org/10.33448/rsd-v10i10.18528

Quadro 2. Características do estudo incluído na revisão sistemática.

\begin{tabular}{|c|c|c|c|c|c|c|}
\hline Título & Autor & País/Ano & Idioma & Objetivo & Participantes por grupos & $\begin{array}{l}\text { Principais } \\
\text { resultados }\end{array}$ \\
\hline $\begin{array}{l}\text { Auriculoterapia para } \\
\text { redução de ansiedade e dor } \\
\text { em profissionais de } \\
\text { enfermagem: ensaio clínico } \\
\text { randomizado }\end{array}$ & Kurebayashi et al. & $\begin{array}{c}\text { Brasil } \\
2017\end{array}$ & Inglês & $\begin{array}{c}\text { Avaliar a efetividade } \\
\text { do protocolo auricular } \\
\text { para redução de } \\
\text { ansiedade (protocolo } \\
\text { APPA), dor e } \\
\text { melhoria de qualidade } \\
\text { de vida em equipe de } \\
\text { enfermagem de um } \\
\text { hospital. }\end{array}$ & $\begin{array}{c}\text { G1- controle ou sem } \\
\text { intervenção: } 31 . \\
\text { G2 - auriculoterapia com } \\
\text { sementes: } 35 \text {. } \\
\text { G3 - auriculoterapia com } \\
\text { agulhas semipermanentes: } 34 \text {. } \\
\text { G4 - auriculoterapia com } \\
\text { esparadrapo ou placebo: } 33 \text {. }\end{array}$ & $\begin{array}{l}\text { Houve diferença } \\
\text { estatística }(\mathrm{p}<0,05) \\
\text { com melhores } \\
\text { resultados para o G3 } \\
\text { na última avaliação } \\
\text { (d de Cohen } \\
1,08 / 17 \% \text { de } \\
\text { redução da } \\
\text { ansiedade). }\end{array}$ \\
\hline
\end{tabular}

Fonte: Autores.. 
Research, Society and Development, v. 10, n. 10, e153101018528, 2021

(CC BY 4.0) | ISSN 2525-3409 | DOI: http://dx.doi.org/10.33448/rsd-v10i10.18528

Neste ensaio foram convidados 180 funcionários da equipe enfermagem de um hospital beneficente do estado de São Paulo para 10 sessões de auriculoterapia, dos quais 133 concluíram o número de sessões previstas. Os participantes possuíam média de idade de 35,7 anos e 112 (84,2\%) eram do sexo feminino. Em relação à classe profissional, 48 (36,1\%) eram auxiliares de enfermagem; $56(42,1 \%)$ técnicos de enfermagem e $29(21,8 \%)$ enfermeiros. Os locais de atuação desses profissionais foram os setores de hemodinâmica e hemodiálise, 45 (33,8\%), unidade de terapia intensiva e pronto-socorro, 39 (29,3\%), setor de imagem, 23 (17,3\%), e outros setores não citados, 26 (19,6\%); 33 (24,8\%) informaram possuir alguma comorbidade.

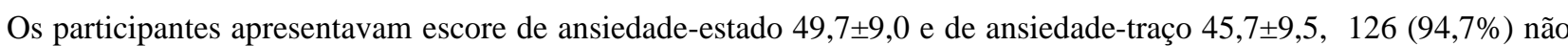
usavam calmantes e dois $(1,5 \%)$ estavam em tratamento psicoterápico. Os profissionais foram aleatoriamente distribuídos em quatro grupos: controle (G1), semente (G2), agulhas semipermanentes (G3) e fita adesiva ou placebo (G4). A intervenção da auriculoterapia consistiu em sessões que ocorreram duas vezes por semana, com duração entre cinco a dez minutos.

A escolha dos acupontos seguiu a indicação do Protocolo Auricular para Dor e Ansiedade - APPA, em sua versão beta, e foi realizada nos seguintes pontos: shenmen, tranquilizante, tálamo, sistema autonômico e ponto zero. Como resultado da pesquisa foi possível observar um efeito moderado na redução da ansiedade nos participantes do G3 de 17\% (d de Cohen = 1,08). A análise do risco de viés está representada na Figura 2, o que demonstra risco de viés incerto em cinco dos sete domínios avaliados.

Figura 2. Análise do risco de viés.

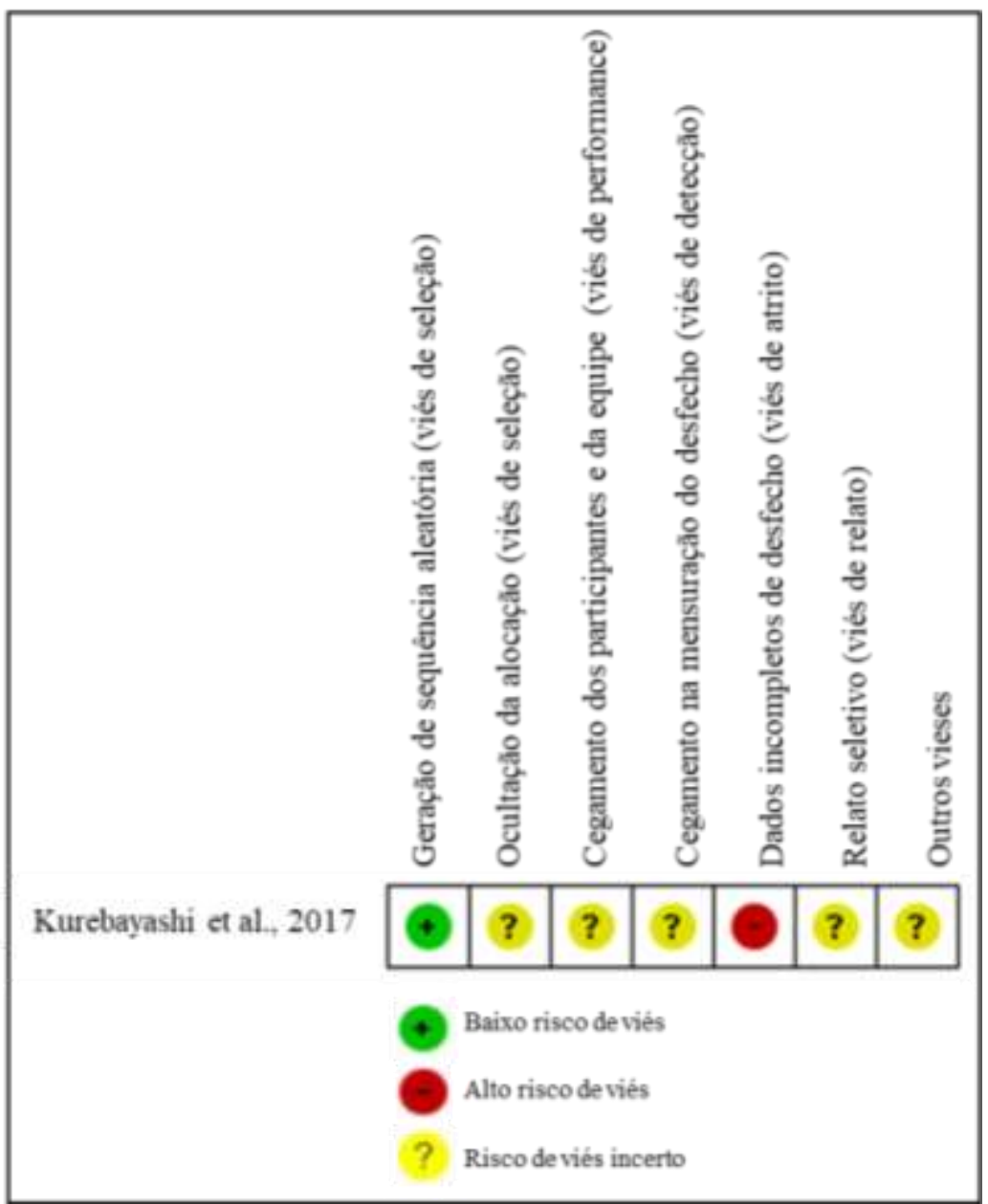

Fonte: Autores. 


\section{Discussão}

A eficácia das práticas integrativas como a auriculoterapia têm sido investigadas para o controle de sintomas de ansiedade na população geral. No entanto, poucos estudos sobre o manejo não farmacológico da ansiedade entre profissionais de saúde têm sido desenvolvidos.

O estudo incluído nesta revisão sugere que a auriculoterapia com agulhas é eficaz para a redução de sintomas de ansiedade entre profissionais de saúde. No entanto, seus resultados precisam ser interpretados com cautela, uma vez que se trata de um ensaio unicêntrico com pequeno tamanho amostral e com risco de viés incerto. A auriculoterapia com agulhas também apresentou resultados satisfatórios na redução dos níveis de estresse e melhoria da qualidade de vida em 175 profissionais de enfermagem de um hospital geral de São Paulo (Kurebayashi \& Silva, 2015).

Estudo de revisão demonstrou que a aplicação da auriculoterapia em indivíduos com transtorno de ansiedade foi eficaz em 78\% dos estudos. Dessa forma, entende-se que a ansiedade é um problema de saúde mental que interfere diretamente no nível de qualidade de vida do indivíduo (Moura et al., 2014).

Os profissionais da saúde constituem um grupo de trabalhadores que compartilham elevada carga física e mental, com exigências de habilidade técnicas e interpessoais, raciocínio clínico, estresse e morte no seu cotidiano. Portanto, são pessoas que estão expostas a experiências que podem impactar negativamente a sua saúde, tais como a ansiedade e o estresse (Minas, Rodacoski \& Sdoukos, 2019; Ribeiro et al., 2020).

A despeito da abrangência das estratégias de busca adotadas, pode-se perceber uma grande carência de estudos primários sobre o tema. Como resultado da busca, um ensaio com profissionais de enfermagem foi incluído na amostra final. No estudo em questão, os pesquisadores implementaram o protocolo APPA, que segue a linha de pensamento da Escola Francesa, por meio da estimulação de pontos corporais no pavilhão auricular, relacionados à sintomatologia (Moura et al., 2014; Moura et al., 2019).

Esse protocolo desenvolvido inicialmente para manejo da ansiedade em populações pobres, com risco de conflitos e desastres, tem como objetivo uniformizar os pontos de aplicação (Corrêa et al., 2020; Jales et al., 2019).

Dessa forma, os pontos-chave que compõem o protocolo APPA são voltados para: ansiedade (shemen), algias em geral (simpático), relaxamento (ponto zero), lombalgias e cervicalgias (tálamo). Esses pontos são adequados para o tratamento da ansiedade, visto que a manutenção do estado ansioso leva a modificações comportamentais, percepção alterada da dor neuropática e incapacidades. Fato que cursa com achados secundários do estudo selecionado, nele obtem-se também como resultado um efeito médio no nível mental da qualidade de vida, com aumento de 13\%, e redução da percepção da dor, em 36\%, no grupo que recebeu o tratamento com agulhas (Artioli, Tavares \& Bertolini, 2019; Pessinoti \& Portnoi, 2016).

Embora tenham sido apresentadas as vantagens da aplicação com sementes, como menor desconforto e possibilidade de ser aplicada por pessoas treinadas para o protocolo, esse tipo de material não promoveu alívio dos sintomas de maneira eficaz, como a auriculoterapia com agulhas. Estudo experimental, prospectivo, randomizado, controlado e simples-cego que teve como objetivo avaliar o efeito clínico da acupuntura auricular nos níveis de ansiedade, demonstrou que a auriculoterapia com agulha semi-permamente é eficaz na diminuição dos níveis de ansiedade, logo após 48 horas da aplicação (Vieira et al., 2018).

Quanto à qualidade metodológica, o ensaio incluído nesta revisão apresentou algumas limitações, como: a dificuldade de adesão dos participantes; o uso de material inadequado para o protocolo, uma vez que o protocolo APPA sugere o uso de material não invasivo; e a ausência de relato sobre o cegamento dos avaliadores do desfecho. De uma forma geral, esses aspectos resultaram em uma avaliação de viés incerto, o que diminui a validade externa dos achados. 
Research, Society and Development, v. 10, n. 10, e153101018528, 2021

(CC BY 4.0) | ISSN 2525-3409 | DOI: http://dx.doi.org/10.33448/rsd-v10i10.18528

\section{Conclusão}

A auriculoterapia pode ser uma importante aliada no controle dos sintomas de ansiedade. Contudo, os resultados desta revisão sistemática não são suficientes para recomendar a sua implementação para o controle dos sintomas de ansiedade em profissionais de saúde. Portanto, são necessárias novas pesquisas multicêntricas que contenham amostras representativas e maior rigor metodológico, a fim de proporcionar evidências sólidas para a construção de protocolos e diretrizes clínicas para o manejo não farmacológico da ansiedade em profissionais de saúde.

\section{Referências}

Artioli, D. P., Tavares, A. L. F. \& Bertolini, G. R. F. (2019). Auriculotherapy : neurophysiology, points to choose, indications and results on musculoskeletal pain conditions : a systematic review of reviews. BrJP, 2(4), 356-61.

Asher, G. N., Jonas, D. E., Coeytaux, R. R., Reilly A. C., Loh Y. L., Motsinger-reif A. A., Winham S. J. (2010). Auriculotherapy for Pain Management : A Systematic Review and Meta-Analysis of Randomized Controlled Trials. J Altern Complement Med, 16(10), 1097-108.

Cardozo-Batista, L., Tucci, A. M. (2020). Effectiveness of an alternative intervention in the treatment of depressive symptoms. J Affect Disord, $276,562-9$.

Carvalho, A. P. V., Silva, V., \& Grande, A. J. (2013). Avaliação do risco de viés de ensaios clínicos randomizados pela ferramenta da colaboração Cochrane. Diagn Tratamento. 18(1), 38-44.

Corrêa, H. P., Moura, C. C., Azevedo, C., Bernardes, M. F. V. G., Mata, L. R. F. P. \& Chianca, T. C. M. (2020). Efeitos da auriculoterapia sobre o estresse, ansiedade e depressão em adultos e idosos: revisão sistemática. Rev Esc Enferm USP, 54, e03626.

Costa, C. O., Branco, J. C., Vieira, I. S., Souza, L. D. M. \& Silva, R. A. (2019). Prevalência de ansiedade e fatores associados em adultos. J Bras Psiquiatria, $68(2), 92-100$.

Dantas, E. S. O. (2021). Saúde mental dos profissionais de saúde no Brasil no contexto da pandemia por Covid-19. Interface - Comunicação, Saúde, Educação, 25, e200203.

Dias, M. O., Souza, N. V. D. O., Penna, L. H. G. \& Gallasch, C. H. (2019). Perception of nursing leadership on the fight against the precariousness of working conditions. Rev Esc Enferm USP, 53, e03492.

Freitas, L. C. S., Sousa, P. H. C. \& Coutinho, B. D. (2020). Auriculoterapia no tratamento da obesidade : uma revisão sistemática. . J Physiother Res, 10(3), $553-565$.

Gao, J., Chen, G., He, H., Liu, C., He, Q., Li, J. \& Wang, J. (2019). The effect of auricular therapy on blood pressure: A systematic review and meta-analysis. Eur J Cardiovasc Nurs, 19(1), 20-30.

Jales, R. D., Gomes, A. L. C., Silva, F. V., Pereira, I. L., Costa, L. F. P. \& Almeida, S. A. (2019). Auriculoterapia no cuidado da ansiedade e depressão. Rev enferm UFPE on line, 13, e240783.

Kurebayashi, L. F. S., Turrini, R. N. T., Souza, T. P. B., Marques, C. F., Rodrigues, R. T. F. \& Charlesworth, K. (2017). Auriculotherapy to reduce anxiety and pain in nursing professionals: a randomized clinical trial. Rev Latino-Am Enfermagem, 25, e2843.

Kurebayashi, L. F. S. \& Silva, M. J. P. (2015). Auriculoterapia chinesa para melhoria de qualidade de vida de equipe de Enfermagem. Rev Bras Enferm, 68(1), $117-23$.

Kwon, C., Lee, B. \& Kim, S. (2020). Effectiveness and safety of ear acupuncture for trauma-related mental disorders after large-scale disasters. Medicine (Baltimore), 99(8), e19342.

Leão, A. M., Gomes, P. I., Ferreira, M. J. M. \& Cavalcanti, L. P. G. (2018). Ansiedade entre estudantes universitários da área da saúde de um grande centro urbano do Nordeste do Brasil. Rev Bras Educ Med, 42(4), 55-65.

Minas, H. O., Rodacoski, G. C. \& Sdoukos, S. S. (2019). Uso de medicamentos psicoativos pelos profissionais de saúde da atenção básica. Revista de Saúde Pública do Paraná, 2, 38-46.

Moura, A., Lunardi, R., Volpato, R., Nascimento, V., Bassos, T. \& Lemes, A. (2018). Fatores associados à ansiedade entre profissionais da atenção básica. Rev Port Enferm Saúde Ment, 19, 17-26.

Moura, C. C., Chaves, E. C. L., Chianca, T. C. M., Ruginsk, S. G., Nogueira, D.A. \& Iunes, D. H. (2019). Effects of auricular acupuncture on chronic pain in people with back musculoskeletal disorders: a randomized clinical trial. Rev Esc Enferm USP, 53, e03418.

Moura, C. C., Carvalho, C. C., Silva, A. M., Iunes, D. H., Carvalho, E. C. \& Chaves, E. C. L. (2014). Auriculoterapia efeito sobre a ansiedade. Rev cuba enferm, $30(2)$.

Perssinotti, D.M. N., Portoni, A. G. (2016). Psychobehavioral and psychosocial aspects of neuropathic pain patients. Rev Dor. 17(1), S79-84. 
Research, Society and Development, v. 10, n. 10, e153101018528, 2021

(CC BY 4.0) | ISSN 2525-3409 | DOI: http://dx.doi.org/10.33448/rsd-v10i10.18528

Ribeiro, I. A. P., Fernandes, M. A., Rocha, D. M., Silva, J. S., Ribeiro, H. K. P. \& Soares, N. S. A. (2020). Consumo de substâncias psicoativas por trabalhadores de enfermagem: revisão integrativa. Texto context enferm, 29, e20180488.

Silva, D. F. O., Cobucci, R. N., Soares-Rachetti, V. P., Lima, S. C. V. C. \& Andrade, F. B. (2021). Prevalência de ansiedade em profissionais da saúde em tempos de COVID-19 : revisão sistemática com metanálise Prevalence of anxiety among health professionals in times of COVID-19: a systematic review with meta-analysis. Ciênc Saúde Colet, 26(2), 693-710.

Vendemiatti, M., Siqueira, E. S., Filardi, F., Binotto, E. \& Simioni, F. J. (2010). Conflitos na gestão hospitalar: o papel da lideraça. Ciênc saúde coletiva, 15(1), $1301-1314$.

Vieira A., Hinzmann, M., Silva, K., Santos, M. J. \& Machado, J. (2018). Clinical effect of auricular acupuncture in anxiety levels of students prior to the exams: A randomized controlled trial. Eur J Integr Med, 20(2018), 188-192. 